\section{Kompartmentsyndrom nach Tibiafraktur: Liefert das Röntgenbild erste Hinweise?}

\author{
Nach einer Tibiafraktur kann das Röntgenbild möglicherweise wertvolle \\ Hinweise auf ein drohendes Kompartmentsyndrom liefern. Welche Kriterien \\ für diese gefürchtete Komplikation sprechen, haben US-Forscher anhand \\ von knapp 1.000 Frakturen untersucht.
}

V or allem ein Prädiktor hatte in der Studie von Christopher Allmon und Kollegen von der School of Medicine der University of Maryland eine hohe Aussagekraft: das Verhältnis aus Frakturlänge und Gesamtlänge der Tibia. In ihrer retrospektiven Studie stieg das Risiko für ein Kompartmentsyndrom (CS) in dem Maß, wie sich dieser Quotient erhöhte. Für jeden Anstieg um $10 \%$ maßen die Forscher ein um den Faktor 1,67

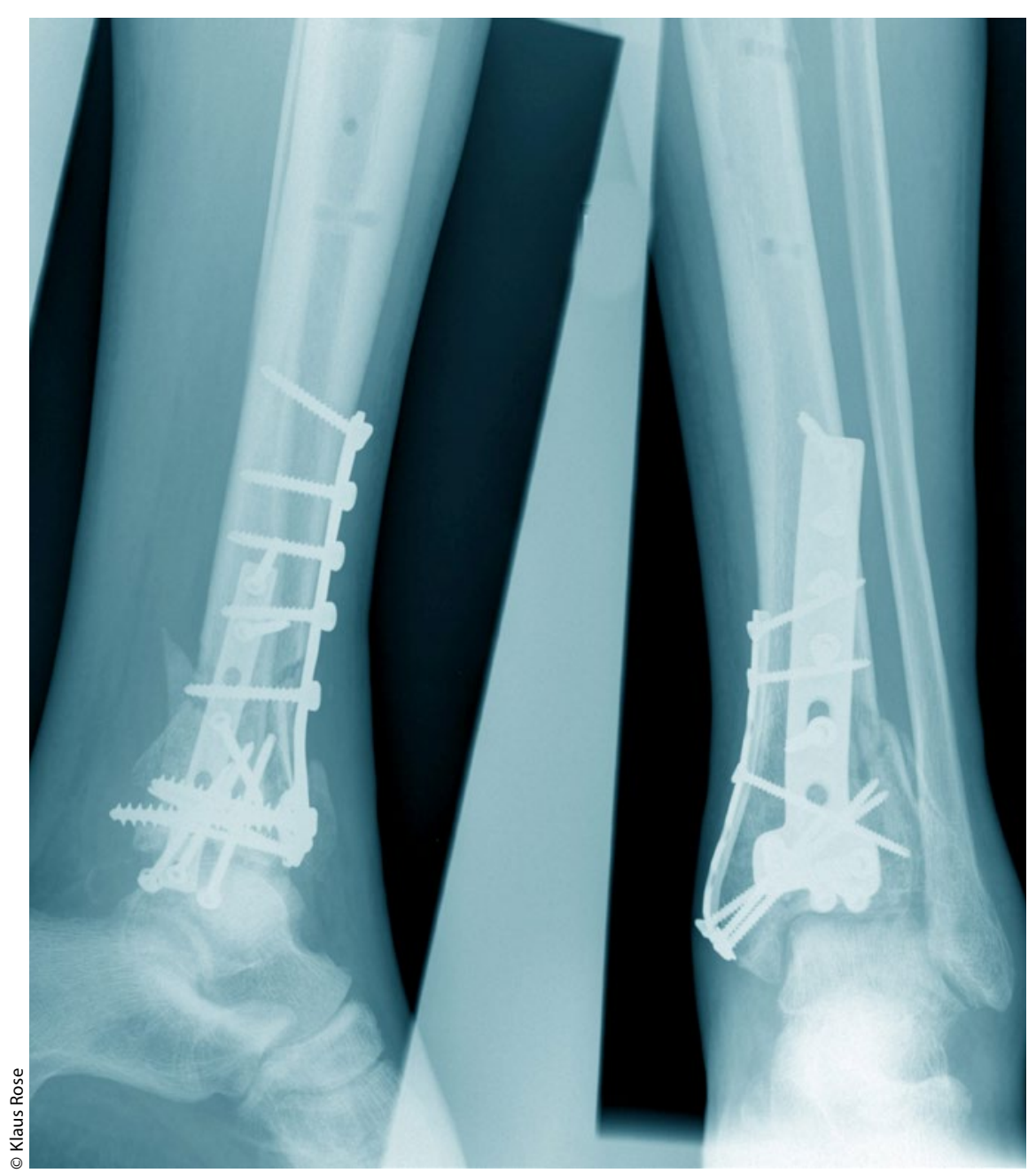

Osteosynthetisch versorgte Tibiafraktur höheres Risiko. Am akkuratesten war die Vorhersage aufgrund dieses Parameters beim Vorliegen von Pilonfrakturen (Odds Ratio, OR 7,09), gefolgt von Brüchen, die das Tibiaplateau involvierten (OR 3,42). Dagegen ergab sich für Tibiaschaftfrakturen in dieser Analyse kein erhöhtes Risiko (OR 1,16).

Insgesamt hatten Allmon und sein Team die Röntgenbilder von 987 Tibiafrakturen ausgewertet. In 56 Fällen (6 \%) hatte sich im Anschluss an die Frakturversorgung ein CS entwickelt. Um statistische Verzerrungen zu vermeiden, hatten die Mediziner jeweils 326 Plateau-, Schaft- und Pilonfrakturen gesammelt und diese Gruppen getrennt ausgewertet. Der jeweilige Anteil mit CS lag bei 12 beziehungsweise 3 oder $2 \%$.

Innerhalb der Plateaufrakturen war das CS-Risiko besonders hoch, wenn die Frakturlänge $20 \%$ oder mehr der Tibialänge betrug. Bei diesen Frakturen spielte außerdem der Schatzker-Typ eine entscheidende Rolle. Patienten mit Schatzker-VI-Frakturen - dabei handelt es sich um eine bikondyläre Fraktur begleitet von einer quer oder schräg verlaufenden Fraktur der Metaphyse - waren am meisten gefährdet: Hier lag die Wahrscheinlichkeit eines CS bei $17 \%$ (von 143 Fällen). Verglichen mit der Referenzgruppe (Schatzker I und II), in der sich die Komplikation nur in $6 \%$ der Fälle entwickelte, war die Risikoerhöhung signifikant (OR 3,46). Auch bei SchatzkerV-Frakturen (bikondyläre Fraktur) kam es in $17 \%$ der Fälle zu einem CS; die Signifikanzschwelle wurde hier allerdings knapp verfehlt $(p=0,06)$. Die Gruppe beinhaltete außerdem nur 24 Fälle.

Ein weiterer Risikoindikator ist den Autoren zufolge das Vorliegen einer begleitenden Fibulafraktur. Dies gelte allerdings nur bei Tibiaplateau-, nicht aber bei Tibiaschaftfrakturen. Da bei den Pilonfrakturen in allen Fällen die Fibula mit betroffen war, lässt sich für diese Gruppe hierzu keine Angabe machen.

Fazit: Warum Pilon- und Plateaufrakturen für ein CS prädisponieren, Schaftfrakturen aber nicht, lässt sich den $\mathrm{Au}$ toren zufolge nur schwer erklären. Möglicherweise liegt es daran, dass im Schaft oft schon geringere Kräfte ausreichen, um eine längere Fraktur zu bewirken. Bei den Schaftfrakturen habe sich außerdem der Marknagel als Behandlungsverfahren etabliert. Dadurch würden Blutungen, die ein CS begünstigen, minimiert.

\section{Dr. Elke Oberhofer}

O'Toole R et al. Radiographic Predictors of Compartment Syndrome Occurring after Tibial Fracture. J Orthop Trauma 2016; online10.1097/ BOT.0000000000000565 